\title{
Interactive Motion-Assisted Exposure Therapy for Veterans with Treatment-Resistant Posttraumatic Stress Disorder: A Randomized Controlled Trial
}

\author{
Marieke J. van Gelderen ${ }^{a, b}$ Mirjam J. Nijdam ${ }^{a, c}$ Joris F.G. Haagen ${ }^{d}$ \\ Eric Vermetten ${ }^{b, e, f}$ \\ ${ }^{a} A R Q$ Centrum'45, Diemen, The Netherlands; ${ }^{b}$ Department of Psychiatry, Leiden University Medical Center, \\ Leiden, The Netherlands; ' Department of Psychiatry, Amsterdam University Medical Centers, Amsterdam, \\ The Netherlands; ${ }^{\mathrm{d}} \mathrm{ARQ}$ Centre of Expertise Impact, Diemen, The Netherlands; ${ }^{\mathrm{e}} \mathrm{ARQ}$ National Psychotrauma Centre, \\ Diemen, The Netherlands; ${ }^{\mathrm{f}}$ Military Mental Health Research, Ministry of Defense, Utrecht, The Netherlands
}

\section{Keywords \\ Posttraumatic stress disorder - Psychotherapy outcome research - Veterans - Treatment-resistant sample · Randomized controlled trial - Treatment outcome · Psychological trauma $\cdot$ Movement $\cdot$ Virtual reality}

\begin{abstract}
Background: Veterans with posttraumatic stress disorder (PTSD) tend to benefit less from evidence-based treatments than other PTSD populations. A novel virtual reality and motion-assisted exposure therapy, called 3MDR, provides treatment in an immersive, personalized and activating context. Objective: To study the efficacy of 3MDR for veterans with treatment-resistant PTSD. Method: In a randomized controlled trial ( $n=43$ ) 3MDR was compared to a non-specific treatment component control group. Primary outcome was clinician-rated PTSD symptoms at baseline, after 3MDR, and at the 12-week and 16-week follow-up (primary end point). Intention-to-treat analyses of covariance and mixed models were applied to study differences between groups at the end point and over the course of intervention, controlling for baseline scores. Results: The decrease in PTSD symptom severity from baseline to end point was significantly greater for
\end{abstract}

3MDR as compared to the control group, with a large effect size $(F[1,37]=6.43, p=0.016, d=0.83)$. No significant between-group difference was detected in the course of PTSD symptoms during treatment when including all time points. The dropout rate was low (7\%), and $45 \%$ of the patients in the 3MDR group improved clinically. The number needed to treat was 2.86. Conclusions: In this trial, 3MDR significantly decreased PTSD symptoms in veterans with, on average, a history of 4 unsuccessful treatments. The low dropout rate may be indicative of high engagement. However, a lack of significant differences on secondary outcomes limits conclusions that can be drawn on its efficacy and underlines the need for larger phase III trials. These data show emerging evidence for 3MDR and its potential to progress PTSD treatment for veterans (Dutch Trial Register Identifier: NL5126).

(c) 2020 The Author(s)
Published by S. Karger AG, Basel

\section{Introduction}

Across North Atlantic Treaty Organization (NATO) partners, tremendous effort has been put towards the preparation and mental health care of servicemen [1]. Despite these efforts, a significant number of veterans

$\begin{array}{ll}\text { karger@karger.com } & \text { C 2020 The Author(s) Karger } \\ \text { www.karger.com/pps } & \text { Published by S. Karger AG, Basel Open caccess } \\ \text { This article is licensed under the Creative Commons Attribution- } & \text { NonCommercial-NoDerivatives 4.0 International License (CC BY- } \\ \text { NC-ND) (http://www.karger.com/Services/OpenAccessLicense). } \\ \text { Usage and distribution for commercial purposes as well as any dis- } \\ \text { tribution of modified material requires written permission. }\end{array}$

Marieke Jeltje van Gelderen

ARQ Centrum'45

Nienoord 13

NL-1112XE Diemen (The Netherlands)

m.van.gelderen@centrum45.nl 
develop posttraumatic stress disorder (PTSD) following deployment, with varying prevalence rates (e.g. UK 3-6\%, USA $4-17 \%$, the Netherlands $5-8 \%[2-4]$ ). PTSD in veterans is typically a chronic disabling condition that is associated with impaired family functioning, lower life satisfaction, unemployment and social exclusion [5-8]. Compared to other mental health issues in veterans, PTSD creates the highest economic burden for society [9]. PTSD treatment is less effective for veterans than other PTSD populations; effect sizes reported in meta-analyses are smaller for veterans, and up to two-thirds retain their PTSD diagnosis following guideline-recommended treatments [10-12]. Treatment dropout ranges between 20 and $40 \%$ in trials with veterans and is considerably higher in routine practice $[13,14]$. This is troublesome, as withdrawal from treatment likely reflects low engagement and predicts poor treatment outcome [15]. Active behavioral and emotional involvement in treatment may be particularly difficult for veterans due to therapy-related barriers (e.g., lack of fit with therapy rationale) and high avoidance during treatment $[13,16]$. Avoidance can lead to suboptimal traumatic memory retrieval, which, in turn, can limit therapeutic effects of exposure therapy [17]. Therefore, increasing behavioral and emotional engagement in treatment could be a fruitful strategy for improving its efficacy.

A novel intervention, multi-modular motion-assisted memory desensitization and reconsolidation (3MDR), was developed to improve outcomes for veterans who did not sufficiently benefit from evidence-based treatments [18]. It combines known therapeutic techniques of virtual reality and eye movement desensitization and reprocessing therapy (EMDR) in a novel context in which patients walk on a treadmill while interacting with personal trauma-related images and music. Several theories provided a rationale as to why $3 \mathrm{MDR}$ was expected to improve engagement and treatment outcomes for this population. Firstly, walking proved to have positive effects on fear extinction and associative thinking $[19,20]$. Secondly, embodied cognition studies provided support for decreased avoidance by walking towards trauma-related images: several studies found that physically approaching a feared object resulted in more positive appraisals of that object, and that such approach behaviors improved exposure therapy results [21-24]. Thirdly, virtual reality is thought to increase presence and in-session attention [25, 26], and is presumed to increase immersion in traumarelated images and music which provide strong and direct links to the traumatic memory [27]. Based on these findings, we expected that a combination of virtual reality, walking, trauma-related images and music with exposure could result in lower avoidance and more optimal memory retrieval during treatment. Adequate engagement and memory retrieval would in turn lead to processing and reconsolidating the memory, aided by a dual-attention task similar to the one used in EMDR $[18,28]$. In a first proof of concept veterans with treatment-resistant PTSD (TR-PTSD) positively evaluated the acceptability of 3MDR [29]. Preliminary findings suggested that 3MDR could lead to clinically meaningful changes, which for some patients were not experienced until 4-6 weeks after the intervention $[18,29,30]$.

The current study aimed to test the efficacy of 3MDR for veterans with TR-PTSD in a randomized controlled trial (RCT) comparing 3MDR to a non-specific treatment component control group (NTCC), consisting of treatment without trauma-focused elements. Based on the literature reviewed above, we expected the combined arsenal of therapeutic techniques in $3 \mathrm{MDR}$ to improve treatment outcomes for veterans who did not respond to prior current evidence-based treatment, and we expected that this effect would occur a few weeks after the 3MDR intervention and increase over time $[18,30]$. Therefore, our primary hypothesis was that $3 \mathrm{MDR}$ would significantly decrease PTSD symptoms at the end point, 16 weeks after start of treatment, as compared to the control intervention. In addition, we explored the course of symptoms during treatment at 6,12 and 16 weeks after start of treatment. Based on preliminary findings, we expected to find significant differences in slopes between the groups in favor of 3MDR $[18,29]$.

\section{Method}

Design

This was a multicenter, parallel, single-blind RCT with two treatment arms. The authors assert that all procedures contributing to this work comply with the ethical standards of the relevant national and institutional committees on human experimentation and with the Helsinki Declaration of 1975, as revised in 2008. All procedures were approved by the Medical-Ethical Review Committee of Leiden University Medical Center (approval No.: P14.325). Reporting was done in accordance with the CONSORT guidelines and methodological recommendations by Guidi et al. [31].

\section{Participants}

Treatment-resistant veterans $(n=43)$ were recruited between 2015 and 2018 at two tertiary mental health care institutes in the Netherlands: ARQ Centrum'45 $(n=28)$ and Mental Health Center Beilen $(n=15)$. Inclusion criteria were: age $18-70$, mastery of Dutch language, meeting DSM-5 criteria for PTSD and treatment 
resistance, defined as persisting PTSD diagnosis and lack of improvement in PTSD symptom severity following a full course of individual trauma-focused therapy or repeated failed trials of individual trauma-focused therapy and treatment duration of at least 6 months [32]. Exclusion criteria were kept to a minimum to be able to recruit a representative treatment-resistant sample. Participants were excluded in case of current acute suicidality, inability to walk, psychosis and severe alcohol/substance dependence.

\section{Medication}

Participants were allowed to use psychotropic medication if stable for 4 weeks before entering the trial and were required to keep medication use stable during the course of the trial. Detailed information on current and prior medication is reported in Table 1, and online supplementary Table 1 (for all online suppl. material, see www.karger.com/doi/10.1159/000505977). At each assessment patients were asked about any changes in medication. An alert was inserted in participants' electronic patient files to inform all involved clinicians that this patient was participating in the current trial and no changes in medication could be made besides clinical urgencies. If psychiatrists needed to change medication in case of clinical urgency, they reported this to the first author (M.J.G.).

\section{Outcome Measures}

\section{Clinical Interviews}

The primary outcome was mean change in PTSD symptom severity as assessed with the Clinician-Administered PTSD Scale for DSM-5 (CAPS-5 [33, 34]). The CAPS- 5 consists of 20 items measuring PTSD symptoms in 4 symptom clusters (intrusions, avoidance, alterations in mood/cognitions and hyperarousal). Each question is rated on a 5-point scale, resulting in a total symptom severity score (range: $0-80$ ). For a diagnosis of PTSD at least 1 intrusive symptom, 1 avoidance symptom, 2 mood and cognitive alteration symptoms, 2 hyperarousal symptoms and impairment must be present (score $\geq 2$ ). Preceding the CAPS -5 at baseline, the Life Events Checklist for DSM-5 was administered. This 17 -item questionnaire assesses exposure to traumatic events [35].

Presence of comorbid psychiatric disorders was assessed with the Mini-International Neuropsychiatric Interview-Plus for DSMIV, as the DSM-5 version was not yet available at the start of the study. Its psychometric quality is well established [36].

The Emory Treatment Resistance Interview for PTSD was used to quantify level of treatment resistance to evidence-based psychological and pharmacological treatments [37]. Points for non-response were given based on available evidence for that treatment ( 3 points for high evidence to 0 points for no evidence; range $0-19$ for pharmacological treatments and 0-38 for psychotherapeutic interventions). This new measure has not been validated. However, it is based on a systematic review and currently the only available structured method to quantify treatment resistance in PTSD.

\section{Self-Report Measures}

The PTSD Checklist for the DSM-5 (PCL-5) was used to measure self-reported PTSD symptom severity during the last month [38]. This 20-item questionnaire corresponds to the DSM-5 symptom criteria for PTSD. Each symptom can be rated on a 5-point scale. Psychometric evaluation of this instrument was positive [39].

Trial of 3MDR for Veterans with

Treatment-Resistant PTSD
Symptoms of general anxiety and depression were assessed with the Hospital Anxiety and Depression Scale (HADS), which consists of 14 questions rated on a 4-point scale. Psychometric qualities of this instrument are well established [40].

The Posttraumatic Avoidance Behaviour Questionnaire (PABQ [41]) was used to identify avoidance behavior in daily life. The PABQ consists of 25 items rated on a 4-point scale. It has been found to be a psychometrically sound instrument [41].

Perceptions of social support were measured with the Interpersonal Support Evaluation List with 12 items. Questions are answered on a 4-point scale. Reliability and validity of this instrument are satisfactory [42].

The validated Cantril's Ladder of Life [43] was used to assess life satisfaction. Participants rated current and future expected quality of life on a 10-point scale. Scores of 7 or higher represent positive quality of life, between 4 and 7 reflect medium quality of life and below 4 reflect poor quality of life.

\section{Treatments}

\section{MDR Treatment}

$3 \mathrm{MDR}$ is a manualized intervention developed through collaboration of the Military Rehabilitation Center, Military Mental Health Care, and Motek Medical B.V. (all located in the Netherlands), and was applied as described in the protocol, which can be viewed in the online supplemental material. Six standardized weekly 3MDR sessions of 70-90 min were followed by an optional 10 weekly treatment sessions depending on patients' needs and therapists' indications. 3MDR was administered on a dual-belt treadmill and a synchronized virtual reality environment, which comprised a 180-degree projection on 3 screens by 3 projectors and a surround sound system. The $3 \mathrm{MDR}$ software consisted of a purpose-built environment to walk in, personalized for each patient by projecting images and playing music, selected by patients themselves. For safety reasons, participants wore a harness whilst on the treadmill. A junior psychologist operated hardware and software out of direct sight of the patient.

Prior to $3 \mathrm{MDR}$ patients selected 10-20 images that strongly reminded them of (elements of) their deployment-related traumatic events. These could be from deployment, the Internet or drawings. The therapist guided picture selection and discussed possible avoidance of images where appropriate. Images were arranged by theme and by a score of $0-10$ subjective units of distress given for each image. Seven images were used each session. In most cases these were 7 different images, but images could also be repeated, particularly when subjective units of distress scores for an image remained high. During the course of treatment new images could be added. Patients also chose music: one piece that reminded them of their time of deployment (e.g., music that was played often during that time) and one piece that reminded patients of the here and now (e.g., contemporary music).

Throughout each session, patients walked at their preferred walking speed, whilst the therapist was standing beside them. A session began with a physical and mental warmup, during which the deployment-related music was played. Patients then entered a virtual tunnel at the end of which the first image was projected. Upon approaching the image, a literal description of the picture was requested, followed by recall of the traumatic memory related to the image and associated feelings and bodily sensations. The patients' associations were recorded by the junior psychologist and projected in real time on the screen. Whilst focusing on these 
Table 1. Demographic and clinical characteristics at baseline ${ }^{\mathrm{a}}$

\begin{tabular}{|c|c|c|c|c|c|c|}
\hline \multirow[t]{2}{*}{ Demographic characteristics } & \multicolumn{2}{|c|}{$\begin{array}{l}\text { Total sample } \\
(n=43)\end{array}$} & \multicolumn{2}{|c|}{$\begin{array}{l}\text { Control group } \\
(n=21)\end{array}$} & \multicolumn{2}{|c|}{$\begin{array}{l}\text { 3MDR group } \\
(n=22)\end{array}$} \\
\hline & $n$ & $\%$ & $n$ & $\%$ & $n$ & $\%$ \\
\hline Male & 42 & 97.7 & 21 & 100.0 & 21 & 95.5 \\
\hline \multicolumn{7}{|l|}{ Education } \\
\hline High school or less & 18 & 41.9 & 9 & 42.9 & 9 & 40.9 \\
\hline Some college & 22 & 51.2 & 11 & 52.4 & 11 & 50.0 \\
\hline Bachelor degree or higher & 3 & 7.0 & 1 & 4.8 & 2 & 9.1 \\
\hline Married or partnered & 36 & 83.7 & 19 & 90.5 & 17 & 77.3 \\
\hline Employed & 9 & 20.9 & 4 & 19.0 & 5 & 22.7 \\
\hline \multicolumn{7}{|l|}{ Country of deployment(s) } \\
\hline Bosnia & 20 & 46.5 & 10 & 47.6 & 10 & 45.5 \\
\hline Afghanistan & 17 & 39.5 & 10 & 47.6 & 9 & 40.9 \\
\hline Lebanon & 9 & 20.9 & 4 & 19.0 & 5 & 22.7 \\
\hline Iraq & 3 & 7.0 & 1 & 4.8 & 2 & 9.1 \\
\hline Other & 7 & 16.3 & 1 & 4.8 & 6 & 27.3 \\
\hline Comorbidity & 35 & 81.4 & 17 & 81.0 & 18 & 81.8 \\
\hline Mood disorder & 30 & 69.8 & 16 & 76.2 & 14 & 63.6 \\
\hline Anxiety disorder & 22 & 51.2 & 11 & 52.4 & 11 & 50.0 \\
\hline Alcohol dependence (mild-moderate) & 5 & 11.6 & 2 & 9.5 & 3 & 13.6 \\
\hline Alcohol abuse & 3 & 7.0 & 1 & 5.0 & 2 & 9.1 \\
\hline Other & 4 & 9.3 & 3 & 14.3 & 1 & 4.5 \\
\hline Current psychotropic medication for PTSD & 28 & 65.1 & 11 & 52.4 & 17 & 77.3 \\
\hline SSRIs & 16 & 37.2 & 6 & 28.6 & 10 & 45.5 \\
\hline Benzodiazepines & 11 & 25.6 & 5 & 23.8 & 6 & 27.3 \\
\hline Atypical antipsychotics & 9 & 20.9 & 4 & 19.0 & 5 & 22.7 \\
\hline Other antidepressants & & 14.0 & 3 & 14.3 & 3 & 13.6 \\
\hline SNRIs & 4 & 9.3 & 1 & 4.8 & 3 & 13.6 \\
\hline Prior trauma-focused treatment for $\mathrm{PTSD}^{\mathrm{b}}$ & 43 & 100.0 & & & & \\
\hline EMDR & 42 & 97.7 & 20 & 95.2 & 22 & 100.0 \\
\hline TF-CBT (including imaginal exposure) & 29 & 67.4 & 12 & 57.1 & 12 & 54.5 \\
\hline TF-CBT group & 18 & 41.9 & 10 & 47.6 & 8 & 36.4 \\
\hline Prior psychotropic medication use & 38 & 88.4 & 17 & 81.0 & 21 & 95.5 \\
\hline SSRI & 27 & 62.8 & 11 & 52.4 & 16 & 72.7 \\
\hline Benzodiazepines & 19 & 44.2 & 11 & 52.4 & 8 & 36.4 \\
\hline Atypical antipsychotics & 18 & 41.9 & 7 & 33.3 & 11 & 50.0 \\
\hline Other antidepressants & 13 & 30.2 & 6 & 28.6 & 7 & 53.8 \\
\hline Anti-epileptics & 8 & 18.6 & 2 & 9.5 & 6 & 27.3 \\
\hline \multirow[t]{2}{*}{ SNRIs } & 6 & 14.0 & 2 & 9.5 & 4 & 18.2 \\
\hline & mean & SD & mean & SD & mean & SD \\
\hline Age & 42.18 & 9.36 & 41.93 & 9.12 & 42.41 & 9.80 \\
\hline Number of deployments & 1.63 & 0.95 & 1.52 & 0.81 & 1.73 & 1.08 \\
\hline Number of prior psychological treatments for PTSD & 4.16 & 1.72 & 4.33 & 1.68 & 4.00 & 1.77 \\
\hline \multicolumn{7}{|l|}{ Number of prior psychopharmacological } \\
\hline treatments for PTSD & 3.40 & 2.69 & 3.00 & 2.78 & 3.77 & 2.62 \\
\hline Emory Treatment Resistance Interview & 4.74 & 3.97 & 4.29 & 3.27 & 5.18 & 4.56 \\
\hline Clinician-Administered PTSD Scale & 44.70 & 7.26 & 46.67 & 5.88 & 42.82 & 8.06 \\
\hline PTSD Checklist for DSM-5 & 53.78 & 8.33 & 53.57 & 7.31 & 53.98 & 9.37 \\
\hline Posttraumatic Avoidance Behaviour Questionnaire & 43.01 & 10.46 & 42.00 & 9.85 & 43.97 & 11.14 \\
\hline Interpersonal Support Evaluation List (12) & 22.05 & 7.48 & 23.71 & 7.62 & 20.45 & 7.16 \\
\hline Hospital Anxiety and Depression Scale & 27.13 & 4.60 & 26.76 & 4.63 & 27.48 & 4.64 \\
\hline Cantril's Ladder of Life Current & 3.86 & 1.52 & 3.52 & 1.17 & 4.18 & 1.76 \\
\hline Cantril's Ladder of Life Future & 6.88 & 1.45 & 6.55 & 1.80 & 7.21 & 0.93 \\
\hline
\end{tabular}

SSRIs, selective serotonin reuptake inhibitors; SNRIs, serotonin noradrenalin reuptake inhibitors; EMDR, eye movement desensitization and reprocessing therapy; TF-CBT, trauma-focused cognitive behavioral therapy. ${ }^{a}$ No statistically significant differences between treatment groups on any variable. ${ }^{b}$ Treatment initiated. 
phrases, patients were asked to track a ball moving from left to right over the picture with random numbers appearing in the ball at the far end of the picture. Patients were required to call these numbers aloud for $30 \mathrm{~s}$. If more than 7 numbers were missed, the task was extended to $45 \mathrm{~s}$. Once the task was complete, the picture faded, and patients were asked for a subjective units of distress rating. Then the virtual tunnel re-appeared, and the sequence was repeated for 7 times in total. Therapists were instructed to address each image and the associated memory for between 3 and 6 min. After the exposure sequence, patients returned to a neutral virtual environment and listened to their contemporary music, which brought them back to the here and now, whilst receiving positive reinforcement from the therapist. Once off the treadmill, each session was completed with a therapist-led discussion about experiences the patient had during the session. The therapist ensured that the patient was fully present in the here and now, allowed for release of tension after the strenuous activity and discussed the patient's post-session coping.

To assess treatment fidelity, during each session the junior psychologist scored a checklist describing all essential elements of a 3MDR session. During the 10 weeks following $3 \mathrm{MDR}$, participants were allowed to receive other treatments to process any (new) therapeutic material that became available through the $3 \mathrm{MDR}$. There were no restrictions. Therapists were given a checklist to fill out after every session in which they reported on techniques used.

Non-Specific Treatment Component Control Group

An NTCC was the control condition in this trial and consisted of non-trauma-focused treatment during which daily stressors and symptoms were addressed. This type of control condition allowed for comparable attention and human interaction variables and has been deemed appropriate for this phase of research with severely ill participants for whom the best available care was ineffective [31, $44,45]$. In NTCC a team of at least one psychiatrist and one mental health worker delivered treatment as much as deemed necessary for up to 16 weeks. NTCC could consist of case management, medication consults, stabilizing interventions, psychoeducation, systemic interventions and cognitive behavioral therapy without trauma-focused elements such as exposure or cognitive restructuring of distorted cognitions related to traumatic events. NTCC was not manualized but applied as standard in clinical practice and monitored. Therapists received instructions on the elements that could be administered in NTCC. Adherence to the trial protocol was monitored with a checklist for treatment components, which included both trauma-focused and non-trauma-focused elements, and was completed by the therapist after each session. At the end of the trial, control participants were offered 3MDR.

\section{Procedure}

Patients were referred to the trial by their therapist when trauma-focused treatment did not result in PTSD symptom improvement. After referral, individuals received information over the telephone or in person. During the first appointment, written informed consent was obtained. At baseline (T0), eligibility was assessed with clinical interviews, and self-report questionnaires were administered. Eligible participants were randomly allocated in a 1:1 ratio. The random sequence was generated with a computer program and had a block size of 6 . A statistician, without further engagement in the study, created the random sequence list, assigned participants and kept a log file of the assignments.

Trial of 3MDR for Veterans with

Treatment-Resistant PTSD
Assessments were conducted after 6 weeks (T1; post-3MDR), 12 weeks (T2) and 16 weeks (T3; primary end point). During T0 and T3 all interviews and self-report measures were administered. At T1 and T2 only the CAPS, PCL-5, PABQ and HADS were administered.

All treatments were outpatient and delivered by PTSD therapists with training and experience in at least one additional trauma-focused therapy (besides 3MDR). To accommodate patient preferences, we aimed for patients to continue with their original therapist during the study as often as possible. As such, 21 therapists were involved in this trial: 7 therapists delivered the $3 \mathrm{MDR}$ only, 7 delivered NTCC only, and 7 delivered both $3 \mathrm{MDR}$ and NTCC. Each therapist received two half-day training sessions in the 3MDR protocol (van Gelderen, Nijdam and Vermetten: Treatment protocol for the preparation and administration of the 3MDR intervention, 2017, see online suppl. material) and regular supervision. Assessors were trained junior psychologists or master's level psychology students. They received monthly group supervision and individual supervision on demand. Assessors were blind to treatment condition. If blinding was broken, another assessor was assigned to the following assessment. Serious adverse events that could influence the course of the disorder or treatment outcomes were monitored [31]. These were defined as any untoward medical occurrence or effect of the intervention that could amount to significant harm, including suicidal intent, mentalhealth-related hospital admissions, self-harm and development of psychotic symptoms [46].

\section{Statistical Methods}

Based on previously found effects of treatments with similar components (virtual reality exposure therapy for veterans $(d=0.69$ [47]), eye movement desensitization and reprocessing for veterans $(d=1.10[48])$, and movement augmentation for a US community sample $(d=2.65[20])$, we expected that $3 \mathrm{MDR}$ would result in at least a significant, medium-sized $(d=0.50)$ improvement in PTSD symptoms. According to the sample size calculation 34 participants were required to provide $80 \%$ power to detect a medium effect in CAPS- 5 change from T0 to T1, T2 and T3 between 3MDR and NTCC. Because of an estimated $20 \%$ dropout, sample size was set at 40 . The actual sample was slightly larger $(\mathrm{N}=43)$ as several participants were deemed eligible simultaneously towards the end of recruitment.

Baseline demographic and clinical characteristics between $3 \mathrm{MDR}$ and NTCC were compared with $t$ tests for continuous variables and $\chi^{2}$ tests for categorical variables. Point-biserial correlations were calculated between changes in medication and CAPS-5 decrease from T0 to T3 for the full sample and between types of medication most used in the $3 \mathrm{MDR}$ group and CAPS -5 decrease from T0 to T3. Analyses were intention-to-treat. For $7 \%$ of the participants the CAPS-5 end point score was missing. As this is slightly above threshold of $5 \%$ for missing data in an intention-totreat analysis, we conducted multiple imputations, and intentionto-treat means and confidence intervals were reported [49]. The Markov chain Monte Carlo algorithm was used for multiple imputation, and 20 data sets were generated.

To test our primary hypothesis that $3 \mathrm{MDR}$ would lead to a significant decrease in CAPS-5 PTSD symptoms as compared to NTCC from T0 to T3, an analysis of covariance (ANCOVA) was conducted. The ANCOVA tested whether the difference in CAPS5 score between groups diverged from 0 at T3, with baseline CAPS- 


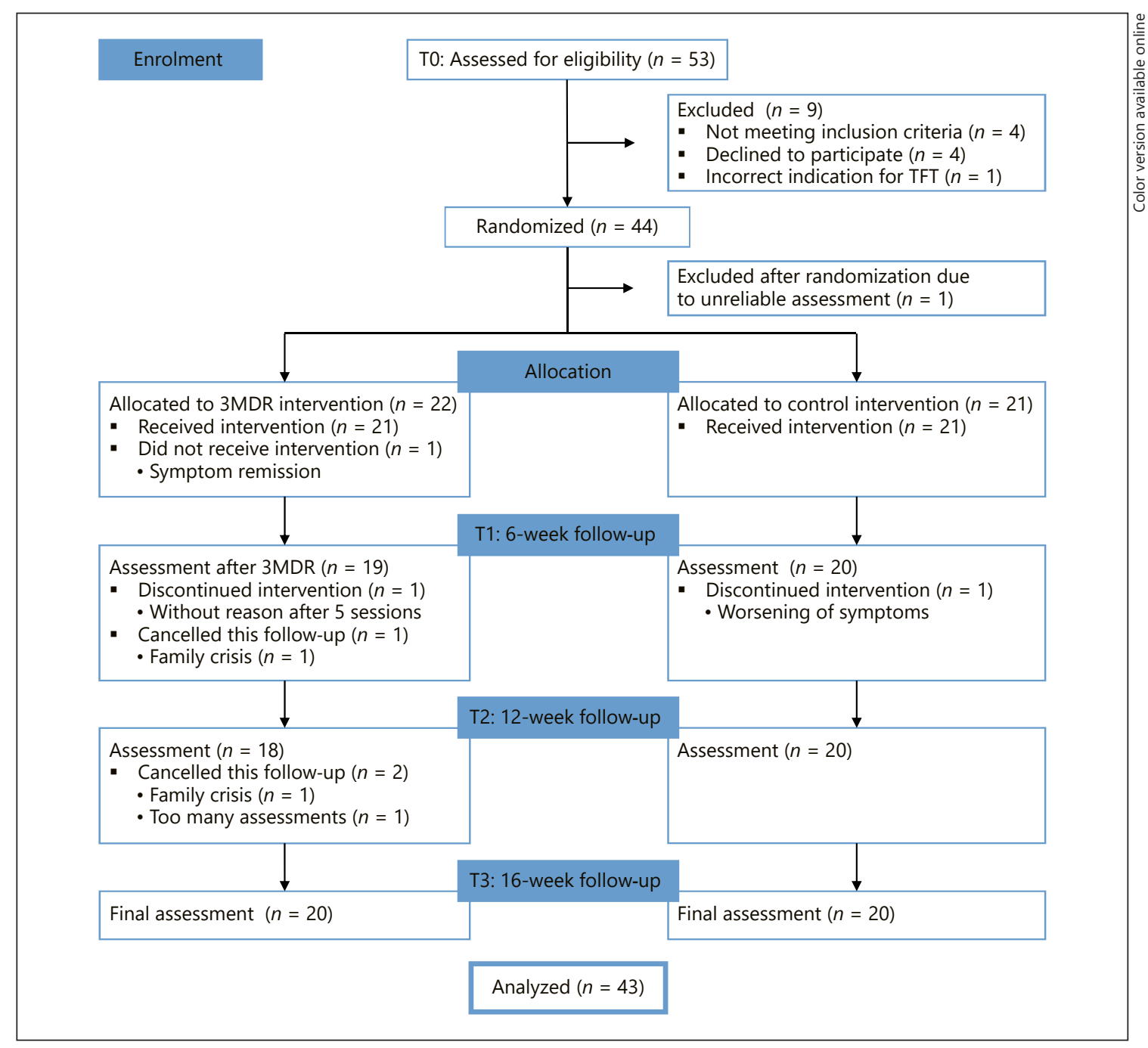

Fig. 1. Participant flow diagram of a randomized controlled trial of 3MDR versus non-trauma-focused treatment. TFT, trauma-focused treatment.

5 score included as covariate. Similarly, differences between groups at T3 were tested with ANCOVAs for secondary outcome measures (PCL-5, PABQ, HADS, Interpersonal Support Evaluation List with 12 items, Cantril's Ladder of Life Current, and Cantril's Ladder of Life Future), with baseline scores included as covariate.

To study differences between conditions in the course of PTSD symptoms during the intervention as measured with the CAPS-5, linear mixed models were applied. Score at T3 was modelled as a function of group (two levels), time (three levels), baseline score, and the interaction between time and intervention. An autoregressive covariance structure for measurements within an individual was applied. If a significant interaction effect was found, differences between groups at T1 and T2 were tested. Likewise, differences between groups in the course of secondary outcomes during the intervention were tested with mixed linear models for secondary outcome measures (PCL-5, PABQ and HADS). Cohen's $d$ effect sizes were calculated. $p$ values of less than 0.05 were considered statistically significant, and two-tailed tests were used throughout.
Participants were categorized as either improved (responders), remained stable, or deteriorated (the latter two categories were considered non-responders) based on individual change in CAPS-5 score from $\mathrm{T} 0$ to $\mathrm{T} 3$. We calculated the reliable change index between both measurement time points and used this as a clinically relevant margin [50]. Using the pooled variance at baseline $(\mathrm{SD}=7.26)$ and test-retest reliability $(r=0.78)$, participants with a positive change of 10 or more points on the CAPS- 5 were defined as responders.

\section{Results}

\section{Participants}

No significant differences were detected at baseline between the 3MDR and NTCC groups on demographic or clinical characteristics (Table 1). The index traumatic 
Fig. 2. PTSD symptom pattern in $3 \mathrm{MDR}$ and control group. Linear mixed modelbased mean CAPS- 5 scores, with CAPS- 5 score at T0 included as covariate, and revised for intention-to-treat. Including standard error bars. The 3MDR intervention was given between T0 and T1. Higher scores indicate more severe PTSD symptoms. * Reflects a significant difference between groups from baseline to end point at 16 weeks (ANCOVA: $F[1,37]=6.43, p=$ $0.016)$.

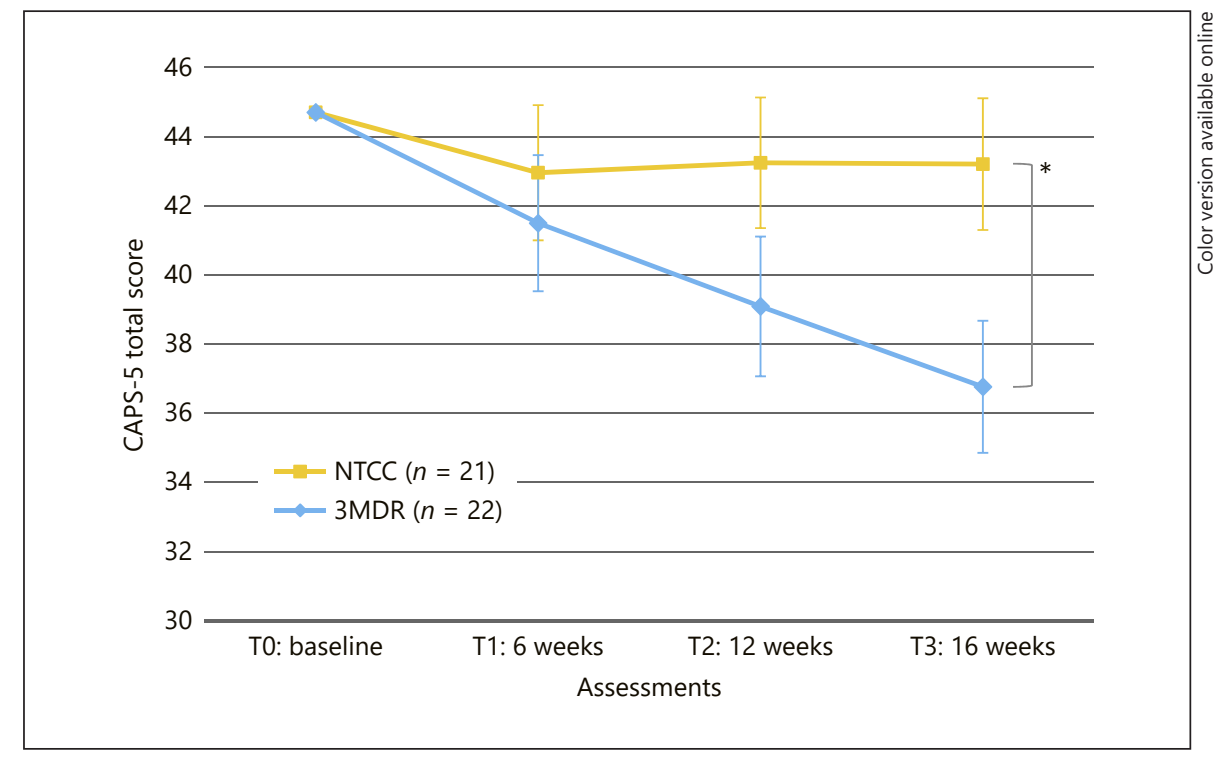

events took place during deployment and were: assault with a weapon $(9,20.9 \%)$, explosion or fire $(7,16.3 \%)$, combat or exposure to a war zone $(6,14.0 \%)$, death caused to someone else $(5,11.6 \%)$, severe human suffering $(5$, $11.6 \%)$, sudden violent death $(4,9.3 \%)$, sudden unexpected death and transportation accident (3, 7.0\%), lifethreatening illness or injury $(2,4.7 \%)$, captivity $(1,2.3 \%)$ and transportation accident (1,2.3\%). Participants, on average, received 4.16 prior psychological treatments and 3.40 prior pharmacological treatments. Three participants withdrew from the study (7.0\%): 1 patient from NTCC (4.7\%) and 2 patients from 3MDR (9.1\%; see CONSORT flow chart in Fig. 1). One participant was excluded after randomization because validity of the assessments was judged to be extremely poor by the assessor and thereby deemed invalid. With respect to safety, no adverse events related to $3 \mathrm{MDR}$ and NTCC were reported, and data did not reveal any increase in suicidal ideations, psychotic symptoms or self-harm nor any additional admissions at mental health care facilities.

\section{Treatment Integrity}

Treatment dosage was comparable across groups: the average amount of treatment hours did not significantly differ between groups (3MDR: mean $=22.27, \mathrm{SD}=13.49$; NTCC: mean $=20.35$, SD $=22.79 ; t(28,95)=-0.32, p=$ $0.75)$ nor did the amount of weeks with a treatment session (3MDR: mean $=10.56, \mathrm{SD}=2.66$; NTCC: mean $=8.74$, $\mathrm{SD}=5.27 ; t(26)=-1.37, p=0.18)$. Treatment sessions following 3MDR most often consisted of case management $(n=20)$, medication consults $(n=6)$, stabilizing interven-

Trial of 3MDR for Veterans with

Treatment-Resistant PTSD tions $(n=9)$ and psychodynamic therapy $(n=4)$. One participant received protocolized evidence-based trauma-focused therapy following the 3MDR intervention. 3MDR treatment adherence was checked for a random selection of $15 \%$ of $3 \mathrm{MDR}$ treatment sessions and proved to be high with a percentage of $91 \%$ of intended therapeutic elements in the selected sessions. NTCC consisted mostly of case management $(n=15)$, medication consults $(n=12)$, stabilizing interventions $(n=11)$, psychoeducation $(n=10)$ and cognitive behavioral therapy without trauma-focused elements $(n=9)$. Checking all sessions showed that adherence to the protocol in NTCC was violated only once: one patient in the control group received one therapy session with trauma-focused elements.

\section{Medication}

Six participants changed psychotropic medication during the trial ( 3 in 3MDR, 3 in NTCC), of which 1 participant discontinued medication (online suppl. Table 1). There were no significant correlations between medication change and difference in PTSD symptom severity from baseline to end point (online suppl. Table 1). Use of benzodiazepines $(r=-0.010, p=0.70)$, selective serotonin reuptake inhibitors $(r=0.19, p=0.43)$ or atypical antipsychotics $(r=-0.37, p=0.11)$ was not significantly correlated with change in CAPS- 5 score from baseline to T3 in the $3 \mathrm{MDR}$ group.

\section{Primary Outcome}

Results of the intention-to-treat analyses for CAPS-5 are shown in Figure 2 and described in Table 2. The pri- 


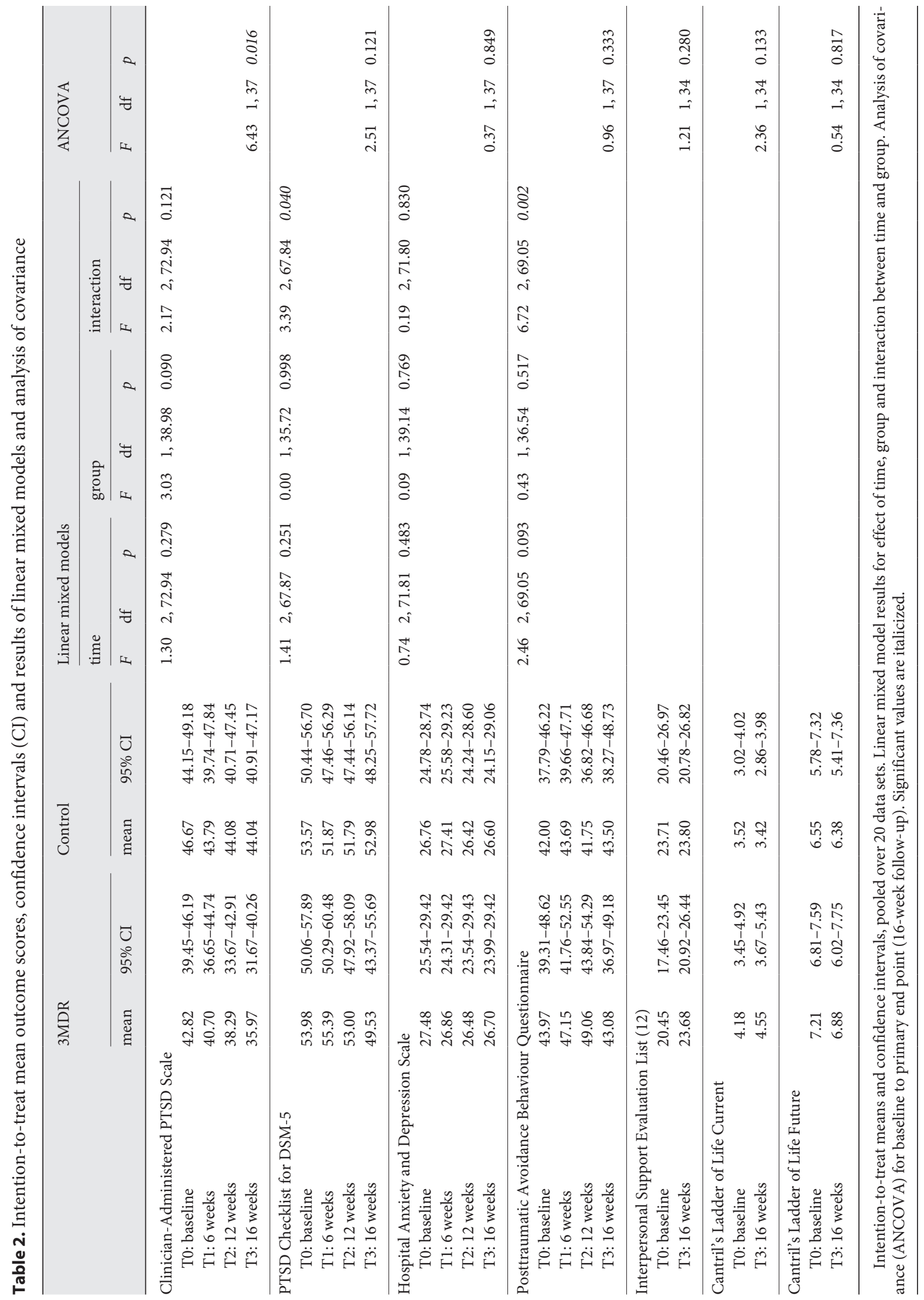


mary ANCOVA demonstrated that participants in the $3 \mathrm{MDR}$ group had significantly greater improvements in CAPS-5 scores at T3 as compared to participants in the NTCC group, when controlling for baseline scores $(F[1$, $37]=6.43, p=0.016)$. CAPS-5 scores decreased 6.60 points more from $\mathrm{T} 0$ to $\mathrm{T} 3$ in the $3 \mathrm{MDR}$ group than in the NTCC group (95\% CI $=1.33-11.87$ ), corresponding to a large effect size $(d=0.83)$ and indicating a positive treatment effect for the 3MDR group. Linear mixed models did not show a significant effect of time $(F[2,72.94]=$ $1.30, p=0.279)$, group $(F[1,38.98]=3.03, p=0.090)$ or interaction between time and group $(F[2,72.94]=2.17$, $p=0.121)$, indicating that the course of CAPS-5 PTSD symptom severity during intervention did not significantly differ between groups when including all postassessment time points.

In the 3MDR group clinically relevant improvement on the CAPS-5 from T0 to T3 was demonstrated in 9 patients ( $45 \%$ responders). Ten patients remained stable, and 1 patient deteriorated (55\% non-responders). In the control group, 2 patients improved (10\%), 18 patients remained stable (90\%) and none of the patients deteriorated. Zero patients in the control group and 2 patients $(10 \%)$ in the $3 \mathrm{MDR}$ group lost their diagnosis from T0 to T3. Rates of clinically meaningful improvement correspond to a number needed to treat of 2.86 , which indicates that 3 veterans with TR-PTSD needed to receive $3 \mathrm{MDR}$ treatment for at least 1 patient to show clinically meaningful improvement on the CAPS-5.

\section{Secondary Outcomes}

Outcomes at all post-assessment time points are presented in Table 2 for all measures. The ANCOVAs did not demonstrate significant differences at $\mathrm{T} 3$ between groups at any of the secondary outcome measures. Linear mixed model analysis revealed a significant interaction effect of group and time for the PCL-5, suggesting differences in course of self-reported PTSD symptoms between groups. Further exploration of the mixed models did not demonstrate a significant difference at $\mathrm{T} 1$ or $\mathrm{T} 2$. This reflects that significant differences between groups could not be demonstrated at separate post-assessments on this measure, even though the interaction effect indicated that a small initial increase in PCL-5 scores was followed by an ongoing decline in the 3MDR group whereas in the NTCC group scores remained at the same level. Similar results were found for the PABQ scores: linear mixed models demonstrated a significant interaction effect, but no significant differences between groups at T1 or T2. The interaction effect showed that mean scores initially in-

Trial of 3MDR for Veterans with

Treatment-Resistant PTSD creased in the $3 \mathrm{MDR}$ group from baseline to T1 and returned to just below baseline level at T3, whilst scores remained at the same level in the NTCC group.

\section{Discussion}

In this RCT of 43 veterans with TR-PTSD, the 3MDR intervention led to significant improvements in PTSD symptom severity as compared to the control condition from pre-treatment to the primary end point at 16 weeks with a large effect size. Dropout was substantially below the usual rates for guideline recommended treatments $[13,14]$. Almost half of the patients in the $3 \mathrm{MDR}$ condition demonstrated clinically relevant PTSD symptom improvement. This confirms our primary hypothesis and demonstrates promising effects for $3 \mathrm{MDR}$ in this first trial. However, contrary to our hypothesis the course of PTSD symptom severity during intervention was not found to significantly differ between groups. No significant differences between groups were detected either in quality of life, depression, self-reported PTSD symptoms, daily life avoidance, and perceived social support at the end point of the trial. This limits conclusions that can be drawn on the efficacy of $3 \mathrm{MDR}$ and stresses the need for further studies into its effects.

\section{Interpretation and Comparison to Existing Studies}

Studies that have considered the efficacy of psychotherapeutic interventions following unsuccessful evidencebased PTSD treatment for veterans and other TR-PTSD populations are very scarce [51]. Results of multiple trials with 3,4-methylenedioxymethamphetamine (MDMA)assisted psychotherapy for TR-PTSD were encouraging with large effect sizes [52-55], and deep brain stimulation shows promise as well, with two positive case reports [56]. Considering that the current trial recruited veterans with, on average, 4 prior unsuccessful treatments and demonstrated a large effect size, 3MDR could prove to be a useful non-invasive, non-pharmacological addition to the toolbox of clinicians working with veterans with TR-PTSD. It should be noted that this study cannot address with certainty whether these changes can be attributed to $3 \mathrm{MDR}$ only, as patients received on average 4.56 treatment-asusual contacts during the 10 weeks following $3 \mathrm{MDR}$. However, since only 1 veteran in the $3 \mathrm{MDR}$ group received additional trauma-focused therapy, we deem it unlikely that these follow-up sessions were the main source of effect.

The rationale for $3 \mathrm{MDR}$ was improving engagement and memory processing by combining virtual reality, 
trauma-related stimuli and walking with a dual-attention task. A recent meta-analysis in active duty personnel and veterans with PTSD who were not treatment-resistant, found positive effects for EMDR (0.83) and virtual reality exposure therapy (VRET; 0.43) in comparison to waitlist or treatment as usual, and attrition rates ranging between 16 and $31 \%$ for EMDR and 5 and 44\% for VRET [57]. Compared to these figures, the current study results are encouraging, especially considering the TR-PTSD sample in the current trial. Dropout rate (7\%) was lower than in EMDR and comparable to VRET, and the effect size was comparable to EMDR and higher than VRET. This suggests that the combined arsenal of therapeutic techniques may indeed have added value by increasing engagement and optimizing memory processing for a treatment-resistant sample. However, the current study cannot confirm whether these expected mechanisms of action were indeed at play. A low dropout rate in $3 \mathrm{MDR}$ might also be due to positive attitudes towards treatment, as the belief that the treatment will be effective could enhance retention and engagement $[13,58]$. 3MDR is likely perceived as state-of-the-art, thereby possibly instilling confidence in treatment. Additionally, the low amount of therapy sessions and a clearly defined end point may have contributed to persistence in treatment [12].

Given the effect on clinician-rated PTSD, the absence of a significant difference between groups on any of the secondary outcomes at the end point was unexpected. Apart from there being no effect and large variance in scores relative to the sample size, this could be explained by potentially low sensitivity to change of some outcome measures for this chronically ill population in a relatively short amount of time. Future studies should shed light on how to best assess outcomes in treatment-resistant samples. Self-reported PTSD in the 3MDR group as compared to the NTCC group decreased from baseline to end point with a medium effect size $(d=0.51)$ without reaching statistical significance. This suggests that large variance relative to the sample size may have been an issue. The course of self-reported PTSD symptoms during intervention indicated an ongoing decrease from posttreatment to follow-up in the 3MDR group. This course might be similar to that described for clinician-rated PTSD, but with a delay. Because of the chronic nature of the disorder in our sample, it is possible that patients were not directly aware of changes in their symptoms and noticed this at a later stage than clinicians in structured interviews. Moreover, therapy in itself could have changed patients' internal standard over the course of treatment, resulting in an underestimation of its self-reported effect directly after treatment (a process known as response shift [59]). While not previously used in treatment outcome research, the PABQ scores in the 3MDR group showed a noteworthy course: avoidance in daily life increased until the 12-week follow-up and returned to baseline level at the 16-week follow-up. Possibly, the confrontational nature of $3 \mathrm{MDR}$, during which in-session avoidance is difficult, resulted in an increased need for control (avoidance) outside of the sessions.

With regard to adverse effects, 1 participant showed a deterioration in PTSD symptoms. He had lost a significant other due to suicide during the trial and reported this as the reason for his symptom increase. We, therefore, deem it unlikely that this worsening of symptoms was related to $3 \mathrm{MDR}$. We did not encounter evidence for other iatrogenic effects [60] either. No serious adverse events were found that would relate to short- or long-term changes in the course, characteristics or responsiveness of the disorder, such as increases in suicidal intent, psychotic symptoms or self-harm. We note that 1 participant withdrew from treatment before finishing 6 sessions with unknown reason. Together, these data seem to indicate that 3MDR was an acceptable and safe treatment in the current sample of veterans. However, future trials should consider a broader range of iatrogenic effects in larger samples with longer follow-up periods to determine potential alterations to the course of PTSD.

\section{Strengths and Limitations}

Some limitations should be noted. In the current study sample size was relatively small and variance in scores quite large, which may have resulted in limited statistical power to detect differences between groups in the course of PTSD symptom severity and in secondary outcomes at the end point. Caution is, therefore, required regarding interpretation of the results. Blinding of patients for treatment condition was not possible. Being assigned to receive this innovative treatment could have positively affected treatment expectancies and this in itself could have improved PTSD symptomatology [61]. However, the continued symptom decrease after treatment argues against this being the exclusive effect. The current research group was involved in the development of 3MDR, increasing chances of researcher allegiance effects. The study needs to be replicated by other research groups, which is currently happening in the USA, Canada and the UK. Treatment fidelity of 3MDR was rated by the psychologist who operated $3 \mathrm{MDR}$, which resulted in treatment fidelity information on all sessions. However, our method was more prone to bias compared to indepen- 
dent rating of videotaped sessions. We note that NTCC was not manualized but was monitored over all sessions, and this type of control group was deemed appropriate for this phase of research. 3MDR and control treatment were provided by 21 therapists, which could have resulted in larger treatment variability. However, treatment fidelity was high despite this number of therapists.

Strengths of the current study include the structured assessment of non-response to prior psychological and pharmacological treatment using the Emory Treatment Resistance Interview for PTSD. Although the current study required patients to have received a minimum of 1 evidence-based treatment, in reality patients had a history of on average 4 psychological treatments for PTSD. This study included veterans with a range of comorbid disorders, reflecting the clinical reality for veterans with PTSD and thereby increasing the external validity of the findings [62]. Given the high number of prior treatments and comorbidity in our sample, we deem it probable that our sample will be representative for a wider TR-PTSD veteran sample. However, representativity will likely be limited to those veterans who are open to PTSD treatment and not to those who have given up on treatment. Other strengths included the intention-to-treat analysis, randomization, blinded assessments and high treatment adherence.

\section{Research Implications}

Future phase III trials with larger sample sizes are warranted to provide more insight into the efficacy of $3 \mathrm{MDR}$ and predictors of treatment response. We recommend the inclusion of a manualized specific factors component control group and long-term assessments of functioning in further randomized controlled trials. In future studies, a staging approach could help to recruit stage 4 (treatment-resistant) participants with associated biomarkers, thereby ensuring representativity of the sample [63]. Finally, cost-effectiveness studies are needed to determine whether 3MDR compares favorably with gold standard interventions taking into account treatment length and associated costs.

\section{Conclusions}

This was the first RCT to study a novel psychological intervention that employed walking, exposure to traumarelated cues in a virtual environment and dual-attention processing for veterans with TR-PTSD. The results are promising as $3 \mathrm{MDR}$ significantly decreased PTSD symptoms for veterans with TR-PTSD and dropout was low, suggesting that patients were able to successfully engage in therapy. However, a lack of significant differences on secondary outcomes, which may or may not be due to limited statistical power, restrict judgments that can be made about the intervention. That being said, failed prior treatment can easily lead to demoralization and loss of confidence in therapy, yet 3MDR led to a clinically meaningful breakthrough in symptomatology for almost half of the participants in this study, even after a history of several unsuccessful treatments. As such, 3MDR could prove to be a valuable treatment option for veterans with TR-PTSD.

\section{Acknowledgement}

The authors thank all participants and colleagues who contributed to this study at ARQ Centrum'45, ARQ National Psychotrauma Centre, Leiden University Medical Center, Motek Medical B.V. and Top Referent Traumacentrum (GGZ Drenthe Mental Health Institute). Special thanks go to Niels van der Aa, Hannah Bonnet, Johannes Gijsbers, Dorien de Groot, Rick de Haart, Kirsten Pellemans and Frans Steenbrink for their support.

\section{Statement of Ethics}

This research was approved by the Medical-Ethical Review Committee of Leiden University Medical Center (approval No.: P14.325) and conducted ethically in accordance with the World Medical Association Declaration of Helsinki. All participants gave written informed consent.

\section{Disclosure Statement}

The authors have no conflicts of interest to declare.

\section{Funding Sources}

This project received funding from the Expert Center for Working Conditions and Health, Ministry of Defense, The Hague, the Netherlands, registered under the title "A randomized controlled study of efficacy of multimodular motion-assisted memory desensitization and reconsolidation (3MDR) as a treatment for posttraumatic stress disorder in veterans."

\section{Author Contributions}

M.J.G., M.J.N. and E.V. were involved in designing the study, carrying it out, analysing and interpreting the data and writing the article. J.F.G.H. made substantial contributions to analyzing and interpreting the data and writing the article. 


\section{References}

1 Vermetten E, Greenberg N, Boeschoten MA, Delahaije R, Jetly R, Castro CA, et al. Deployment-related mental health support: comparative analysis of NATO and allied ISAF partners. Eur J Psychotraumatol. 2014 Aug;5(1): 23732.

2 Eekhout I, Geuze E, Vermetten E. The longterm burden of military deployment on the health care system. J Psychiatr Res. 2016 Aug; 79:78-85.

3 Reijnen A, Rademaker AR, Vermetten E, Geuze E. Prevalence of mental health symptoms in Dutch military personnel returning from deployment to Afghanistan: a 2-year longitudinal analysis. Eur Psychiatry. 2015 Feb;30(2):341-6.

4 Richardson LK, Frueh BC, Acierno R. Prevalence estimates of combat-related post-traumatic stress disorder: critical review. Aust N Z J Psychiatry. 2010 Jan;44(1):4-19.

5 Cohen E, Zerach G, Solomon Z. The implication of combat-induced stress reaction, PTSD, and attachment in parenting among war veterans. J Fam Psychol. 2011 Oct;25(5): 688-98.

6 Monson CM, Taft CT, Fredman SJ. Militaryrelated PTSD and intimate relationships: from description to theory-driven research and intervention development. Clin Psychol Rev. 2009 Dec;29(8):707-14.

7 Koenen KC, Stellman SD, Sommer JF Jr, Stellman JM. Persisting posttraumatic stress disorder symptoms and their relationship to functioning in Vietnam veterans: a 14-year follow-up. J Trauma Stress. 2008 Feb;21(1): 49-57.

8 Kintzle S, Keeling M, Xintarianos E, TaylorDiggs K, Munch C, Hassan AM, et al. Exploring the economic and employment challenges facing US veterans: a qualitative study of volunteers of America service providers and veteran clients. California: Centre for Innovation and Research on Veterans and Military Families, University of Southern California, School of Social Work; 2015.

9 Currier JM, Holland JM, Drescher KD. Residential treatment for combat-related posttraumatic stress disorder: identifying trajectories of change and predictors of treatment response. PLoS One. 2014 Jul;9(7):e101741.

10 Watts BV, Schnurr PP, Mayo L, Young-Xu Y, Weeks WB, Friedman MJ. Meta-analysis of the efficacy of treatments for posttraumatic stress disorder. J Clin Psychiatry. 2013 Jun; 74(6):e541-50.

11 Steenkamp MM, Litz BT, Hoge CW, Marmar CR. Psychotherapy for Military-Related PTSD: A Review of Randomized Clinical Trials. JAMA. 2015 Aug;314(5):489-500.

12 Haagen JF, Smid GE, Knipscheer JW, Kleber RJ. The efficacy of recommended treatments for veterans with PTSD: a metaregression analysis. Clin Psychol Rev. 2015 Aug;40:18494.
13 Hoge CW, Grossman SH, Auchterlonie JL, Riviere LA, Milliken CS, Wilk JE. PTSD treatment for soldiers after combat deployment: low utilization of mental health care and reasons for dropout. Psychiatr Serv. 2014 Aug; 65(8):997-1004.

14 Monson CM, Schnurr PP, Resick PA, Friedman MJ, Young-Xu Y, Stevens SP. Cognitive processing therapy for veterans with militaryrelated posttraumatic stress disorder. J Consult Clin Psychol. 2006 Oct;74(5):898-907.

15 Elkin I, Yamaguchi J, Arnkoff D, Glass C, Sotsky S, Krupnick J. "Patient-treatment fit" and early engagement in therapy. Psychother Res. 1999;9(4):437-51.

16 Hundt NE, Ecker AH, Thompson K, Helm A, Smith TL, Stanley MA, et al. "It didn't fit for me:" A qualitative examination of dropout from prolonged exposure and cognitive processing therapy in veterans. Psychol Serv. 2018 Nov; Epub Ahead of Print. https://doi org/10.1037/ser0000316.

17 Zoellner LA, Feeny NC, Bittinger JN, BedardGilligan MA, Slagle DM, Post LM, et al. Teaching trauma-focused exposure therapy for PTSD: critical clinical lessons for novice exposure therapists. Psychol Trauma. 2011; 3(3):300-8.

18 Van Gelderen MJ, Nijdam MJ, Vermetten E. An innovative framework for delivering psychotherapy to patients with treatment-resistant posttraumatic stress disorder: rationale for interactive motion-assisted therapy. Front Psychiatry. 2018 May;9:176.

19 Oppezzo M, Schwartz DL. Give your ideas some legs: the positive effect of walking on creative thinking. J Exp Psychol Learn Mem Cogn. 2014 Jul;40(4):1142-52.

20 Powers MB, Medina JL, Burns S, Kauffman BY, Monfils M, Asmundson GJ, et al. Exercise augmentation of exposure therapy for PTSD: rationale and pilot efficacy data. Cogn Behav Ther. 2015;44(4):314-27.

21 Koch SC. Rhythm is it: effects of dynamic body feedback on affect and attitudes. Front Psychol. 2014 Jun;5:537.

22 Woud ML, Becker ES, Rinck M. Implicit evaluation bias induced by approach and avoidance. Cogn Emotion. 2008;22(6):1187-97.

23 Price TF, Harmon-Jones E. Embodied emotion: the influence of manipulated facial and bodily states on emotive responses. Wiley Interdiscip Rev Cogn Sci. 2015 Nov-Dec;6(6): 461-73.

24 Wolitzky KB, Telch MJ. Augmenting in vivo exposure with fear antagonistic actions: a preliminary test. Behav Ther. 2009 Mar;40(1): $57-71$.

25 Rizzo A, Reger G, Gahm G, Difede J, Rothbaum BO. Virtual reality exposure therapy for combat-related PTSD. In: Shiromani PJ, Keane TM, LeDoux JE, editors. Post-traumatic stress disorder: basic science and clinical practice. Totowa: Humana Press; 2009. p. 375-99.
26 Krijn M, Emmelkamp PM, Olafsson RP, Biemond R. Virtual reality exposure therapy of anxiety disorders: a review. Clin Psychol Rev. $2004 \mathrm{Jul} ; 24(3): 259-81$.

27 Gersons B, Meewisse M, Nijdam M, Olff M. Protocol: brief eclectic psychotherapy for posttraumatic stress disorder (BEPP). Amsterdam: Academic Medical Centre, University of Amsterdam; 2011.

28 Sack M, Zehl S, Otti A, Lahmann C, Henningsen $\mathrm{P}$, Kruse J, et al. A comparison of dual attention, eye movements, and exposure only during eye movement desensitization and reprocessing for posttraumatic stress disorder: results from a randomized clinical trial. Psychother Psychosom. 2016;85(6):357-65.

29 Vermetten E, Meijer L, van der Wurff P, Mert A. The effect of military motion-assisted memory desensitization and reprocessing treatment on the symptoms of combat-related post traumatic stress disorder: first preliminary results. Stud Health Technol Inform. 2013;191:125-7.

30 Nijdam MJ, Vermetten E. Moving forward in treatment of posttraumatic stress disorder: innovations to exposure-based therapy. Eur J Psychotraumatol. 2018 May;9(1):1458568.

31 Guidi J, Brakemeier EL, Bockting CL, Cosci F, Cuijpers P, Jarrett RB, et al. Methodological recommendations for trials of psychological interventions. Psychother Psychosom. 2018; 87(5):276-84.

32 National Collaborating Centre for Mental Health. Post-traumatic stress disorder: The management of PTSD in adults and children in primary and secondary care. Leicester, United Kingdom: Gaskell; 2005.

33 Weathers F, Blake D, Schnurr P, Kaloupek D, Marx B, Keane T. The clinician-administered PTSD scale for DSM-5 (CAPS-5). Interview available from the National Center for PTSD at www.ptsd.va.gov 2013.

34 Weathers FW, Bovin MJ, Lee DJ, Sloan DM, Schnurr PP, Kaloupek DG, et al. The Clinician-Administered PTSD Scale for DSM-5 (CAPS-5): development and initial psychometric evaluation in military veterans. Psychol Assess. 2018 Mar;30(3):383-95.

35 Weathers F, Blake D, Schnurr P, Kaloupek D, Marx B, Keane T. The life events checklist for DSM-5 (LEC-5). Instrument available from the National Center for PTSD at www.ptsd. va.gov 2013.

36 Van Vliet IM, de Beurs E. [The MINI-International Neuropsychiatric Interview. A brief structured diagnostic psychiatric interview for DSM-IV in ICD-10 psychiatric disorders]. Tijdschr Psychiatr. 2007;49(6):393-7. Dutch.

37 Dunlop BW, Kaye JL, Youngner C, Rothbaum B. Assessing treatment-resistant posttraumatic stress disorder: the Emory treatment resistance interview for PTSD (E-TRIP). Behav Sci (Basel). 2014 Dec;4(4):511-27. 
38 Weathers FW, Litz BT, Keane TM, Palmieri PA, Marx BP, Schnurr PP. The PTSD checklist for DSM-5 (PCL-5). Scale available from the National Center for PTSD at www.ptsd. va.gov 2013.

39 Bovin MJ, Marx BP, Weathers FW, Gallagher MW, Rodriguez P, Schnurr PP, et al. Psychometric properties of the PTSD checklist for diagnostic and statistical manual of mental disorders-fifth edition (PCL-5) in veterans. Psychol Assess. 2016 Nov;28(11):1379-91.

40 Bjelland I, Dahl AA, Haug TT, Neckelmann D. The validity of the Hospital Anxiety and Depression Scale. An updated literature review. J Psychosom Res. 2002 Feb;52(2):69-77.

41 van Minnen A, Hagenaars MA. Avoidance behaviour of patients with posttraumatic stress disorder. Initial development of a questionnaire, psychometric properties and treatment sensitivity. J Behav Ther Exp Psychiatry. 2010 Sep;41(3):191-8.

42 Merz EL, Roesch SC, Malcarne VL, Penedo FJ, Llabre MM, Weitzman OB, et al. Validation of interpersonal support evaluation list12 (ISEL-12) scores among English- and Spanish-speaking Hispanics/Latinos from the HCHS/SOL Sociocultural Ancillary Study. Psychol Assess. 2014 Jun;26(2):384-94.

43 Atkinson T. The stability and validity of quality of life measures. Soc Indic Res. 1982;10(2): 113-32.

44 Gold SM, Enck P, Hasselmann H, Friede T, Hegerl U, Mohr DC, et al. Control conditions for randomised trials of behavioural interventions in psychiatry: a decision framework. Lancet Psychiatry. 2017 Sep;4(9):725-32.

45 Mohr DC, Spring B, Freedland KE, Beckner V, Areán P, Hollon SD, et al. The selection and design of control conditions for randomized controlled trials of psychological interventions. Psychother Psychosom. 2009;78(5): 275-84.

46 Parry GD, Crawford MJ, Duggan C. Iatrogenic harm from psychological therapies - time to move on. Br J Psychiatry. 2016;208(3): $210-2$.

47 DiMauro J. Exposure therapy for posttraumatic stress disorder: a meta-analysis. Mil Psychol. 2014;26(2):120-30.
48 Goodson J, Helstrom A, Halpern JM, Ferenschak MP, Gillihan SJ, Powers MB. Treatment of posttraumatic stress disorder in U.S. combat veterans: a meta-analytic review. Psychol Rep. 2011 Oct;109(2):573-99.

49 Jakobsen JC, Gluud C, Wetterslev J, Winkel P. When and how should multiple imputation be used for handling missing data in randomised clinical trials - a practical guide with flowcharts. BMC Med Res Methodol. 2017 Dec;17(1):162-71.

50 Jacobson NS, Truax P. Clinical significance: a statistical approach to defining meaningful change in psychotherapy research. J Consult Clin Psychol. 1991 Feb;59(1):12-9.

51 Koek RJ, Schwartz HN, Scully S, Langevin JP, Spangler S, Korotinsky A, et al. Treatmentrefractory posttraumatic stress disorder (TRPTSD): a review and framework for the future. Prog Neuropsychopharmacol Biol Psychiatry. 2016 Oct;70:170-218.

52 Mithoefer MC, Mithoefer AT, Feduccia AA, Jerome L, Wagner $M$, Wymer $J$, et al. 3,4-methylenedioxymethamphetamine (MDMA)-assisted psychotherapy for posttraumatic stress disorder in military veterans, firefighters, and police officers: a randomised, double-blind, dose-response, phase 2 clinical trial. Lancet Psychiatry. 2018 Jun;5(6):48697.

53 Ot'alora G M, Grigsby J, Poulter B, Van Derveer JW 3rd, Giron SG, Jerome L, et al. 3,4-Methylenedioxymethamphetamine-assisted psychotherapy for treatment of chronic posttraumatic stress disorder: A randomized phase 2 controlled trial. J Psychopharmacol. 2018 Dec;32(12):1295-307.

54 Mithoefer MC, Wagner MT, Mithoefer AT, Jerome L, Doblin R. The safety and efficacy of $\{+/-\} 3,4$-methylenedioxymethamphetamineassisted psychotherapy in subjects with chronic, treatment-resistant posttraumatic stress disorder: the first randomized controlled pilot study. J Psychopharmacol. 2011 Apr;25(4):439-52.
55 Oehen P, Traber R, Widmer V, Schnyder U. A randomized, controlled pilot study of MDMA ( \pm 3,4-Methylenedioxymethamphetamine)-assisted psychotherapy for treatment of resistant, chronic Post-Traumatic Stress Disorder (PTSD). J Psychopharmacol. 2013 Jan;27(1):40-52.

56 Koek R, Langevin J, Krahl S, Chen J, Sultzer $\mathrm{D}$, Mandelkern $\mathrm{M}$, et al. Basolateral amygdala deep brain stimulation for treatment refractory combat PTSD: data from the first two cases. Brain Stimul. 2019;12(2):429-30.

57 Kitchiner NJ, Lewis C, Roberts NP, Bisson JI. Active duty and ex-serving military personnel with post-traumatic stress disorder treated with psychological therapies: systematic review and meta-analysis. Eur J Psychotraumatol. 2019 Nov; 10(1):1684226.

58 Graham B, Garcia NM, Burton MS, Cooper AA, Roy-Byrne PP, Mavissakalian MR, et al. High expectancy and early response produce optimal effects in sertraline treatment for post-traumatic stress disorder. Br J Psychiatry. 2018 Dec;213(6):704-8.

59 Schwartz CE, Sprangers MA. Guidelines for improving the stringency of response shift research using the thentest. Qual Life Res. 2010 May;19(4):455-64.

60 Fava GA, Rafanelli C. Iatrogenic Factors in Psychopathology. Psychother Psychosom. 2019;88(3):129-40

61 Zoellner LA, Roy-Byrne PP, Mavissakalian M, Feeny NC. Doubly randomized preference trial of prolonged exposure versus sertraline for treatment of PTSD. Am J Psychiatry. 2019 Apr;176(4):287-96.

62 Skodol AE, Schwartz S, Dohrenwend BP, Levav I, Shrout PE, Reiff M. PTSD symptoms and comorbid mental disorders in Israeli war veterans. Br J Psychiatry. 1996 Dec;169(6): $717-25$.

63 McFarlane AC, Lawrence-Wood E, Van Hooff M, Malhi GS, Yehuda R. The Need to Take a Staging Approach to the Biological Mechanisms of PTSD and its Treatment. Curr Psychiatry Rep. 2017 Feb;19(2):10.
Trial of 3MDR for Veterans with

Treatment-Resistant PTSD
Psychother Psychosom 2020;89:215-227 DOI: $10.1159 / 000505977$ 